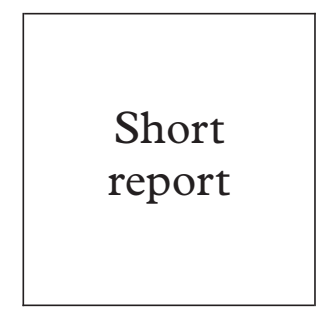

\title{
Inferior vena cava filters for HIV infected patients with pulmonary embolism and contraindications to anticoagulation
}

\author{
Maryam Shahmanesh, Joe Brooks, Penny J Shaw, Robert F Miller
}

Objectives: To describe the mode of presentation, interventions, and outcome of HIV infected
patients with pulmonary embolism and a contraindication to anticoagulation, who were treated with a bird's nest filter.

Methods: Retrospective review of case records and imaging department database at UCL Hospitals, London, UK.

Results: Three patients had pulmonary embolism and contraindications to anticoagulation. Contraindications were concomitant intracerebral pathology in two patients (one also had bleeding from gastric Kaposi's sarcoma and the other was cognitively impaired with HIV associated dementia complex) and alcohol induced liver disease/binge drinking in the third patient. Anticoagulation was avoided by introducing a bird's nest filter into the inferior vena cava via the common femoral vein. During follow up (7, 8, and 21 months) no complications or recurrent pulmonary emboli occurred.

Conclusion: The bird's nest inferior vena cava filter has a role in preventing further pulmonary emboli in HIV infected patients with contraindications to anticoagulation.

(Sex Transm Inf 2000;76:395-397)

Keywords: pulmonary embolism; HIV; AIDS; haemorrhage; anticoagulation

\section{Introduction}

In the general population pulmonary embolism is a common potentially fatal condition that is often overlooked in life. ${ }^{12}$ Pulmonary emboli are responsible for $10 \%$ of all deaths in hospital and are a contributory factor in an additional $10 \%{ }^{1}$ In contrast, reports of venous thromboembolism in patients with HIV infection are sparse. ${ }^{34}$ Anticoagulation is first line therapy for venous thromboembolism and detailed guidelines have been created. ${ }^{5}$ The major complication is haemorrhage which is especially likely from focal lesions, such as peptic ulcers and tumours. ${ }^{6}$ Oral anticoagulation with warfarin may be problematic in patients with erratic heavy alcohol intake, cognitive impairment, and in patients taking other medication as there is potential for drug-drug interactions. ${ }^{78}$

If anticoagulation is contraindicated, or has caused complications, alternative primary therapy is interruption of the inferior vena cava using a metal filter. This technique was originally described by Greenfield et al and involves percutaneous placement of the filter in the inferior vena cava via the femoral vein under fluoroscopic control. ${ }^{5910}$ Newer modified filters such as the "bird's nest" filter are also effective, with low rates of recurrent pulmonary embolism $(<3 \%) .^{5}{ }^{10-12}$ This report describes successful use of the bird's nest filter in three HIV infected patients with venous thromboembolism in whom there were contraindications to anticoagulation.

\section{Patients}

CASE 1

A 34 year old white HIV positive ex-injection drug using man was admitted in November
1998 with a history of 3 months of increasingly poor memory, 2 weeks of a painful, swollen left calf, and 1 week of recurrent trivial haemoptyses without chest pain or dyspnoea. Examination revealed a left calf deep vein thrombosis (DVT), confirmed by ultrasound, with thrombus limited to the popliteal vein. The chest was clinically clear but a chest radiograph showed left mid-zone peripheral consolidation, typical of pulmonary infarction.

The patient had presented 5 months previously when HIV infection was diagnosed; at this time his CD 4 count was 40 cells $\times 10^{6} / 1$ and viral load 70900 copies/ml. He began primary Pneumocystis carinii pneumonia prophylaxis with cotrimoxazole and highly active antiretroviral therapy (HAART) with zidovudine, lamivudine, and nelfinavir. Two weeks later he presented with a 2 day history of severe generalised headache; cranial magnetic resonance imaging (MRI) showed a single ring enhancing lesion with surrounding oedema in the right parietal lobe. A thallium-201 scan showed uptake ratio of 3.27 , strongly suggesting lymphoma. Whole brain radiotherapy was given.

Repeat cranial MRI in November 1998 showed a reduction in size of the mass and surrounding oedema and widespread white matter signal abnormality in both cerebral hemispheres; this was thought to represent HIV leucoencephalopathy. Systemic anticoagulation was contraindicated because of the risk of haemorrhage into the cerebral lesion, and because of concerns about drug compliance in the light of the cognitive impairment and cranial MRI abnormalities. A bird's nest (Cook, Bloomington, IN, USA) filter was 


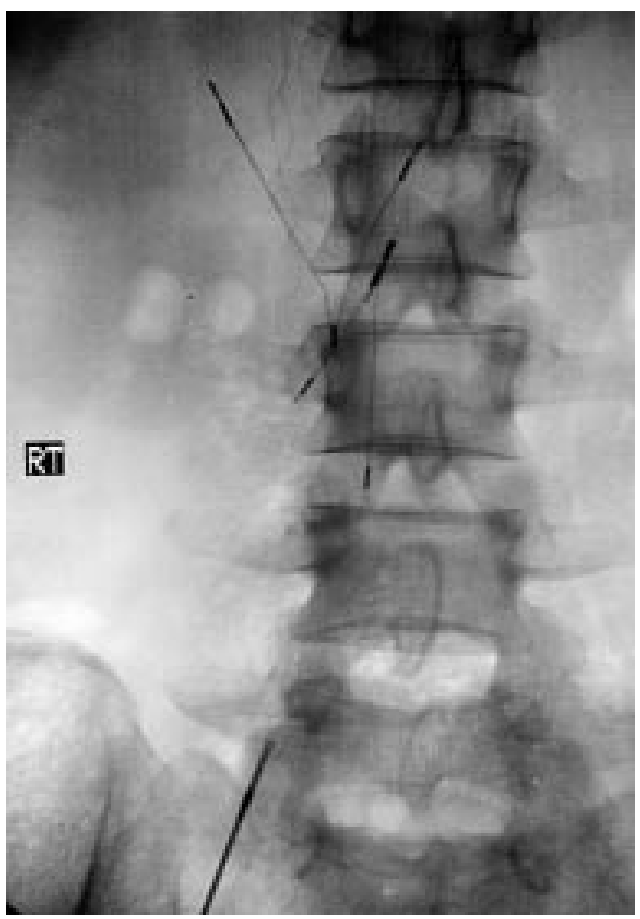

Figure 1 Case 1. Radiograph showing bird's nest filter in place in inferior vena cava. The filter consists of two $V$-shaped stainless steel semirigid struts which have at each end a tiny hook to aid fixation to the wall of the inferior vena cava. The struts are attached to four $0.18 \mathrm{~mm}$ diameter stainless steel wires, each $25 \mathrm{~cm}$ long. The wires are preshaped and randomly bent in many different directions to produce a crisscrossing tangle - the bird's nest. Only the V-shaped struts are visible.

introduced percutaneously via the right common femoral vein into the inferior vena cava and placed below the renal veins (fig 1). The calf DVT was treated with a compression stocking. The patient remains moderately cognitively impaired and taking HAART under close supervision 21 months later with no further episodes of venous thromboembolism and without signs of pulmonary hypertension.

CASE 2

A 35 year old heterosexual African woman presented in May 1999 with pulmonary tuberculosis. At this time HIV infection was also diagnosed. She was treated with quadruple antituberculosis therapy but declined HAART. She subsequently presented with rifampicin induced thrombocytopenia, which resolved when the drug was withdrawn, and a gastrointestinal bleed. Endoscopy showed extensive gastric Kaposi's sarcoma. The patient was intolerant of cyclical vincristine with bleomycin and so was treated with liposomal doxyrubicin. Recurrent anaemia caused by blood loss from gastric Kaposi's sarcoma was treated with blood transfusion. In early November 1999 the CD4 count was 200 cells $\times 10^{6} / 1$ and viral load $>500000$ copies $/ \mathrm{ml}$ and the patient began zidovudine, lamivudine, and nelfinavir.

In mid November 1999 the patient presented with a 10 day history of headache and a 3 day history of left sided pleuritic chest pain. During examination she had a generalised tonic-clonic seizure. There was no focal neurological deficit but there was a left basal pleural rub. A chest radiograph showed bilateral coarse parenchymal infiltrates due to Kaposi's sarcoma. A computerised tomographic (CT) pulmonary angiogram demonstrated filling defects in segmental arteries in the left upper lobe, diagnostic of pulmonary emboli. A cranial CT scan showed multiple ring enhancing masses with surrounding oedema and midline shift. Antitoxoplasma therapy was begun with sulphadiazine and pyrimethamine, and sodium valproate was started. Anticoagulation was contraindicated because of the risk of bleeding with gastric Kaposi's sarcoma and the cerebral lesions. A bird's nest filter was inserted. After 3 weeks of antitoxoplasma therapy a repeat cranial CT scan showed marked reduction in size of the lesions and surrounding oedema. The patient continues on antituberculosis therapy, antitoxoplasma therapy, valproate, HAART, and cyclical doxorubicin. Apart from transfusion dependent anaemia she is well after 8 months of follow up; she has had no further episodes of venous thromboembolism and has no signs of pulmonary hypertension.

\section{CASE 3}

A 32 year old white homosexual man was admitted in December 1999 with inability to care for himself, protracted vomiting, and haematemesis. Emergency endoscopy showed marked gastritis. The patient had been HIV antibody positive for 16 years; the most recent CD 4 count was 20 cells $\times 10^{6} / 1$ and viral load 80200 copies $/ \mathrm{ml}$. The patient had discontinued HAART after many months of poor compliance. He was known to be hepatitis B immune and to have chronic alcohol dependency with frequent "binge" drinking.

Examination revealed multiple spider naevi, palmar erythema, and ascites but no hepatosplenomegaly. Investigations showed $\mathrm{Hb} 8.2$ $\mathrm{g} / \mathrm{dl}$, platelets $25 \times 10^{9} / 1$, ALT five times the upper limit of normal, albumin $25 \mathrm{~g} / \mathrm{l}$ and, despite intravenous vitamin $\mathrm{K}$, the prothrombin time was 17 seconds, and international normalised ratio 1.5. The patient was transfused with blood. The ascites increased, an ascitic tap was sterile, and so frusemide and spironolactone were begun. Ten days after admission the patient complained of sudden onset of left sided pleuritic pain and a non-productive cough. A chest radiograph showed "plate" atelectasis at the left lung base and a CT pulmonary angiogram was diagnostic of pulmonary emboli.

Anticoagulation was contraindicated because of the history of poor compliance with HAART and erratic heavy alcohol consumption. A bird's nest inferior vena cava filter was inserted. After 7 months of follow up, he has had no further episodes of venous thromboembolism and has no signs of pulmonary hypertension.

\section{Discussion}

The three patients reported here all had contraindications to anticoagulation and were at high risk of further pulmonary emboli. There were also potential drug-drug interactions between warfarin and sulphadiazine in 
our second case, ${ }^{7}$ and in the third case, between spironolactone and warfarin, ${ }^{7}$ so bird's nest caval filters were used. None of our patients experienced complications from filter insertion or had further pulmonary emboli. Complications of the bird's nest filter in the general population include recurrent pulmonary emboli in $1.3-2.7 \%$, inferior vena cava thrombosis in $2.9 \%$, and migration of the filter within the inferior vena cava in $0-0.2 \% .^{11}{ }^{12}$

We were not able to monitor neuroradiological response to antitoxoplasma therapy with MRI in our second case. Although the bird's nest filter is made of material which has low ferromagnetism, the device is not MRI compatible for 2 weeks after placement owing the risk of migration. CT scanning was used for monitoring response and did not compromise patient care.

In summary, in these three HIV infected patients with venous thromboembolism and contraindications to anticoagulation, placement of bird's nest caval filters was safe and prevented recurrent pulmonary emboli. This intervention should be considered in HIV infected patients with venous thromboembolism and contraindications to anticoagulation.

We thank Dr M Raphael for inserting the filter in patient 3, and Louise Hinds for typing the manuscript.
Conflict of interest: none. Source of funding: none.

Contributors: All authors contributed to data collection and to writing drafts of the manuscript. The original proposal was put forward by RM who wrote the first and final drafts of the manuscript.

1 Linblad B, Sternby WH, Berquist D. Incidence of venous thromboembolism verified by necropsy over 30 years. $B M \mathcal{F}$ 1991;302:709-11.

2 Anderson FA, Wheeler B, Goldberg RJ, et al. A population based perspective of the hospital incidence and case-fatality rates of deep vein thrombosis and pulmonary embolism. Arch Intern Med 1991;151:933-8.

3 Becker DM, Saunders TJ, Wispelwey B, et al. Venous thromboembolism in AIDS. Am f Med Sci 1992;303:395-

4 Howling SJ, Shaw PJ, Miller RF. Acute pulmonary embolism in patients with HIV disease. Sex Transm Inf 1999;75:25-9.

5 Hyers TM, Hull RD, Weg JG. Anti-thrombotic therapy for venous thromboembolic disease. Chest 1995;108:335S$51 \mathrm{~S}$.

6 Coon Ww, Willis PW. Hemorrhagic complications of anticoagulant therapy. Arch Intern Med 1974;133:386-92.

7 Karalliedde L, Henry J, eds. Anticoagulants. In: Drug interactions. 1st ed. London: Edward Arnold, 561-81.

8 Pozniak AL, Miller RF, Ormerod LP. Treatment of tuberculosis in persons with HIV infection. AIDS 1999;13:435-45.

9 Fink JA, Jones BT. The Greenfield filter as the primary means of therapy in venous thromboembolic disease. Surg Gynecol Obstet 1991;172:253-6.

10 Becker DM, Philbrick JT, Selby B. Inferior vena cava filters. Arch Intern Med 1992;152:1985-94.

11 Roehm JOF, Johnsrude IS, Barth MH, et al. The bird's nest inferior vena cava filter: progress report. Radiology 1988; 168:745-9.

12 Nicholson AA, Ettles OF, Paddon AJ et al. Long-term follow-up of the bird's nest IVC filter. Clin Radiol 1999;54: 759-64. 